DOI: $10.5216 /$ cab.v14i3.6587

\title{
DESEMPENHO DE CABRITOS CASTRADOS TRATADOS COM CALÊNDULA E ASSOCIAÇÕES
}

\author{
SÉrgio Fernandes Ferreira ${ }^{1}$, Erica Bertha FÜHrich Raupp BeZerra de Mello Oliveira ${ }^{2}$, \\ Carlos Elysio Moreira da FonseCA ${ }^{3}$, Maria Ignez Carvalho Ferreira ${ }^{3}$, Mirton José Frota \\ MORENZ $^{4}$ \\ ${ }^{1}$ Pós-graduando da Escola de Veterinária e Zootecnia da Universidade Federal de Goiás, Goiânia, GO, Brasil. \\ sergio-ff@hotmail.com \\ ${ }^{2}$ Pós-graduanda da Universidade Federal Rural do Rio de Janeiro, Seropédica, RJ, Brasil. \\ ${ }^{3}$ Professores Doutores da Universidade Federal Rural do Rio de Janeiro, Seropédica, RJ, Brasil. \\ ${ }^{4}$ Pesquisador Doutor da EMBRAPA Gado de Leite, Juiz de Fora, MG, Brasil
}

O objetivo do presente experimento foi avaliar as respostas de desempenho de caprinos castrados, submetidos a dois tratamentos da ferida cirúrgica: tratamento convencional com pomada à base de óxido de zinco, óleo de pinho, caulim e xilol e spray à base de permetrina, fenoxibenzil e DDVP (diclorvós) e tratamento alternativo com uso de fitoterápico com pomada à base de extrato de Calendula offcinalis e associações. Foram utilizados doze cabritos sem raça definida com peso inicial médio de $15,5 \mathrm{~kg}$, criados em confinamento total, separados em dois grupos dispostos em delineamento inteiramente casualizado para constituir dois tratamentos com seis repetições cada, visando comparar o desempenho dos animais tratados de forma convencional em relação ao tratamento com extrato de Calendula offcinalis e associações. Empregada a técnica de orquiectomia (castração) por abertura da bolsa escrotal e com cordão espermático descoberto no dia 0 , os dois grupos foram tratados até a completa cicatrização da ferida cirúrgica no $51^{\circ}$ dia de experimentação. As medidas biométricas analisadas foram ganho de peso $(\mathrm{kg})$, ganho de altura de cernelha $(\mathrm{cm})$, ganho de altura de garupa $(\mathrm{cm})$, ganho de perímetro torácico $(\mathrm{cm})$ e escore de condição corporal. Os resultados foram analisados pelo pacote estatístico SAEG e teste de Tukey $(\mathrm{P}>0,05)$. Não houve diferença significativa entre os tratamentos $(\mathrm{P}>0,05)$ para nenhum dos parâmetros analisados. Todas as características biométricas, à exceção do escore corporal, aumentaram no período de avaliação. Não houve impacto negativo no uso de fitoterápico em relação às características observadas.

PALAVRAS-CHAVE: caprinos; castração; desempenho; fitoterápico.

\section{PERFORMANCE OF CASTRATED KID GOATS TREATED WITH CALENDULA AND ASSOCIATIONS}

\section{ABSTRACT}

The purpose of this experiment was to evaluate the performance responses of castrated goats, submitted to two surgical wound treatments: conventional treatment with ointment made of zinc oxide, pine oil, kaolin and xylene and permethrin, phenoxybenzyl and DDVP spray (dichlorvos) and alternative phytotherapic treatment using herbal medicine with ointment made of Calendula offcinalis extract and associations. Twelve undefined breed goats with an average initial weight of $15.5 \mathrm{~kg}$, raised in total confinement, were separated into two groups and arranged in a completely randomized design to constitute two treatments with six replicates each, in order to compare the performance of animals treated conventionally and with Calendula offcinalis extract and associations. We employed the orchiectomy (castration) technique by opening the scrotum and spermatic cord on day 0 , and both groups were treated until complete healing of the surgical wound on the $51^{\text {st }}$ day of trial. We analyzed the following biometric parameters: weight gain $(\mathrm{kg})$, withers height gain $(\mathrm{cm})$, croup height gain $(\mathrm{cm})$, thotacic perimeter gain $(\mathrm{cm})$ and body condition score. The results were analyzed using the Statistical Package SAEG and 
Tukey's test $(\mathrm{P}>.05)$. There was no significant difference between treatments $(\mathrm{P}>0.05)$ for any of the parameters analyzed. All biometric characteristics, with the exception

KEYWORDS: castration; goats; growth; phytotherapy.

\section{INTRODUÇÃO}

A criação de caprinos está distribuída por todo o território brasileiro, sendo que, das 558 microrregiões do Brasil, 552 têm algum registro de caprinos. Esses dados mostram que, de alguma forma, a criação destes pequenos ruminantes faz parte do cotidiano de quase a totalidade dos municípios brasileiros (MARTINS \& GARAGORRY, 2007).

Nas áreas rurais, a caprinocultura constitui uma atividade sócio-econômica de destaque, sendo uma importante fonte proteico-alimentar (carne e leite), bem como fonte de renda, por meio da venda de couro e do excedente de carne (MADRUGA et al., 2000).

A criação de caprinos no Brasil é desenvolvida principalmente por pequenos produtores que, normalmente, contam com pouca tecnologia, sofisticação e com baixo investimento em infraestrutura, ignorando práticas simples de controle e desempenho e desconhecendo fontes alternativas para o manejo.

Uma das práticas de manejo adotadas durante a fase de cria é a castração de machos até o $3^{\circ}$ mês de vida. Segundo BRAGA et al. (2003), a prática de castração é realizada pelos criadores com o objetivo de favorecer a expressão das potencialidade dos animais e, com isso, obter uniformidade da carcaça em termos de qualidade, conservação e aspecto da carne de animais castrados destinados ao abate. Também há a facilidade de manejo dos machos, que se tornam mais dóceis e sem o odor, provocado pelas glândulas sexuais, característico dos animais inteiros, que prejudica a comercialização da carne.

Existem vários métodos de castração. $\mathrm{O}$ método cirúrgico, burdizzo e anel de borracha são os mais conhecidos. O método cirúrgico é o mais seguro e mais difundido entre os criadores (COSTA, 2007). Entretanto, a forma e os produtos com os quais os animais são tratados após a castração e durante o período de cicatrização vão influenciar no desenvolvimento natural de atividades fisiológicas básicas e no desempenho.

No Brasil, existem centenas de plantas com reconhecidas propriedades medicinais que são of body condition, increased during the evaluation period. There was no negative impact of the use of herbal medicine on the characteristics observed.

usadas pela população de forma empírica; contudo, por falta de pesquisas comprovando a eficácia do seu uso, acabam desacreditadas ou simplesmente não são aceitas como alternativa à falta de recursos ou quando o uso de alopáticos é vedado, como na agropecuária orgânica, em que se utilizam recursos da homeopatia e fitoterapia.

O uso de plantas medicinais é uma opção à alopatia em casos de pequenas alterações do rebanho (LANS et al., 2007). Algumas plantas, tanto nativas quanto exóticas e com potencial agronômico, são utilizadas para este fim.

Um desses tratamentos alternativos é o uso de extratos de calêndula (Calendula officinalis) e associações, que formam uma pomada contendo calêndula, capim citronela (Cimbopogom nardus), neem (Azadiractha indica), cera de abelha (Apis mellifera L.) e própolis. A C. officinalis é uma planta de origem europeia, usada no tratamento de feridas, empregada na forma dinamizada ou na forma de tintura-mãe nas formulações de pomadas e cremes (CAMPOS et. al, 2000; LANS et. al, 2007). A C. officinalis tem sido bastante utilizada em produtos dermatológicos, com ação antiinflamatória. Sua utilização é maior nos casos de queimaduras, contusões, cortes e erupções, sendo considerada de uso seguro e aplicada topicamente diversas vezes ao dia (GUTERRES et al., 2002).

O objetivo deste estudo é avaliar o desempenho de caprinos castrados, submetidos ao tratamento alternativo da ferida cirúrgica com pomada contendo extrato de Calendula offcinalis e associações e ao tratamento convencional com pomada à base de óxido de zinco, óleo de pinho, caulim e xilol e spray à base de permetrina, fenoxibenzil e DDVP.

\section{MATERIAL E MÉTODOS}

O presente estudo foi conduzido no Setor de Caprinos da Fazenda do Instituto de Zootecnia (FAIZ) da Universidade Federal Rural do Rio de Janeiro, Município de Seropédica, no período de 25 de março a 14 de maio de 2007. O Município de Seropédica (região metropolitana) está localizado na Baixada Fluminense, sudoeste do Estado do Rio de Janeiro, a $43^{\circ} 41^{\prime}$ de longitude 
Oeste e $22^{\circ} 46^{\prime}$ de latitude Sul.

Os animais foram manejados em sistema de confinamento total e receberam água $a d$ libitum, capim elefante (Panicum sp.) duas vezes ao dia e concentrado constituído de $50 \%$ de fubá de milho, $25 \%$ de farelo de trigo e $25 \%$ de farelo de soja, duas vezes ao dia.

Foram utilizados doze animais SRD (sem raça definida) com peso vivo inicial médio de 15,5 $\mathrm{kg}$ (CV 11,18\%), dispostos em delineamento inteiramente casualizado, separados em dois grupos homogêneos, formando então dois tratamentos com seis repetições cada.

Antes de serem castrados, os animais permaneceram em jejum alimentar por 18 horas e em jejum hídrico por 12 horas. Também foram aferidas medidas biométricas de peso, escore de condição corporal (ECC), perímetro torácico, altura de cernelha e altura de garupa.

Foi empregada a técnica de orquiectomia (castração) por duas aberturas na bolsa escrotal e com cordão espermático descoberto no dia 0 . Após esse procedimento, houve o acompanhamento da ferida cirúrgica dos animais, com limpeza com água e sabão de coco e novo curativo aberto a cada três dias para observar a total cicatrização.

O grupo controle foi submetido a tratamento da ferida cirúrgica com pomada à base de óxido de zinco (20\%), óleo de pinho (5\%), caulim $(32 \%)$ e xilol $(6 \%)$ e spray à base de permetrina $(0,28 \%)$ e DDVP $(0,62 \%)$, e o grupo fitoterápico com pomada comercial (Fitoderme laboratório Fitopet) à base de extrato de Calendula officinalis (1\%), oleo de Azadiractha indica (1\%), óleo de Cimbopogom nardus (1\%), própolis $(0,5 \%)$ e cera de abelha (2\%), até a cicatrização completa.

Os parâmetros zootécnicos foram aferidos até a cicatrização completa da ferida cirúrgica do último animal. Foram realizadas pesagens com balança mecânica; medições de perímetro torácico (tomando-se como base o esterno e a cernelha, passando a fita métrica detrás da paleta), de altura de garupa (considerada a distância entre a tuberosidade sacra e a extremidade distal do membro posterior) e de altura da cernelha (considerada a distância entre a região da cernelha e a extremidade distal do membro anterior) com fita métrica; além de aferições de escore de condição corporal (ECC) na escala de 1 a 5 de acordo com método proposto por MORANDFEHR \& HERVIEU (1999), em que 1 corresponde a animal muito magro e 5, a animal obeso, realizadas em intervalos de sete dias.

Os resultados de ganho de peso, ganho em altura de cernelha, ganho em altura de garupa e ganho em perímetro torácico foram submetidos à análise de variância e as médias foram comparadas pelo teste de Tukey, assumindo $5 \%$ de probabilidade de erro. Para a variável ECC, utilizou-se o teste não-paramétrico de KruskalWallis. Foi utilizado auxilio do software estatístico SAEG (2007).

\section{RESULTADOS E DISCUSSÃO}

Os resultados do ganho de peso $(\mathrm{kg})$, ganho de altura de cernelha $(\mathrm{cm})$, ganho de altura de garupa $(\mathrm{cm})$, ganho de perímetro torácico $(\mathrm{cm})$ e perda de escore de condição corporal encontramse na Tabela 1, acompanhado do respectivo erro padrão, para os dois tratamentos, ao final dos 51 dias de experimentação, tempo para completa cicatrização do ferimento causado pela pratica de castração.

Tabela 1. Ganho médio e erro padrão para medidas biométricas e ECC

\begin{tabular}{lcc}
\hline Fonte de & \multicolumn{2}{c}{ Média + Erro Padrão } \\
\cline { 2 - 3 } variação & Convencional & Fitoterápico \\
\hline Ganho de Peso $(\mathrm{kg})$ & $1,42 \pm 0,40^{\mathrm{a}}$ & $1,67 \pm 0,40^{\mathrm{a}}$ \\
Altura de Cernelha $(\mathrm{cm})$ & $4,17 \pm 0,83^{\mathrm{a}}$ & $3,33 \pm 0,67^{\mathrm{a}}$ \\
Altura de Garupa $(\mathrm{cm})$ & $1,83 \pm 0,48^{\mathrm{a}}$ & $3,67 \pm 1,09^{\mathrm{a}}$ \\
Perímetro Torácico $(\mathrm{cm})$ & $0,83 \pm 0,40^{\mathrm{a}}$ & $1,67 \pm 0,56^{\mathrm{a}}$ \\
Escore de Condição Corporal & $-0,75^{\mathrm{a}}$ & $-0,75^{\mathrm{a}}$ \\
\hline
\end{tabular}

Médias, na coluna, seguidas de letras diferentes são diferentes $(\mathrm{P}<0,05)$ pelo teste de Tukey.

Não houve diferença significativa entre os tratamentos $(\mathrm{P}>0,05)$ para nenhum dos parâmetros analisados (Tabela 1). Os cabritos tratados com pomada à base de extrato de Calendula officinalis e associações mostraram desenvolvimento semelhante aos tratados convencionalmente, sem diferença estatística entre os tratamentos em relação às características observadas. 
Avaliar apenas o ganho de peso individualmente ao final do período experimental é precipitado. É razoável pensar que medidas isoladas não sejam suficientes para definir completamente o desempenho animal, pois, como pode ser observado na Tabela 1 e Figura 1, apesar do aumento do peso, altura de cernelha, altura de garupa e perímetro torácico, houve decréscimo do escore de condição corporal.

Segundo RIBEIRO (1997), essa observação pode ser explicada pelo fato de que, apesar de estarem crescendo, os animais também estavam emagrecendo, o que provavelmente foi causado pelo efeito comportamental negativo de dor e estresse que a castração exerce sobre a ingestão de alimentos pelos animais e pelo aumento da demanda energética para atividade celular de multiplicação e renovação na ferida cirúrgica.

Todas as características biométricas, à exceção do escore corporal (Figura 1), aumentaram com idade de avaliação. O mesmo aconteceu no estudo de MENEZES et al. (2007), que estudou diferentes grupos genéticos.

LOUCA et al. (1977) relataram que cabritos machos não castrados cresceram mais rapidamente $\mathrm{e}$ apresentaram maior eficiência alimentar do que cabritos castrados, enquanto que NORMAN (1985) afirmou que a castração geralmente reduz a idade em que a gordura se deposita na carcaça e que caprinos castrados podem produzir carcaças com pontuação de $1-2 \%$ maior do que os não castrados, principalmente quando a castração ocorre antes dos 6 meses.

O ganho de peso dos animais aumentou, mesmo nos primeiros dias após a castração (Figura 2). Esse efeito, aumento do ganho de peso, em animais recém submetidos a manejo que promove estresse não é comumente verificado.

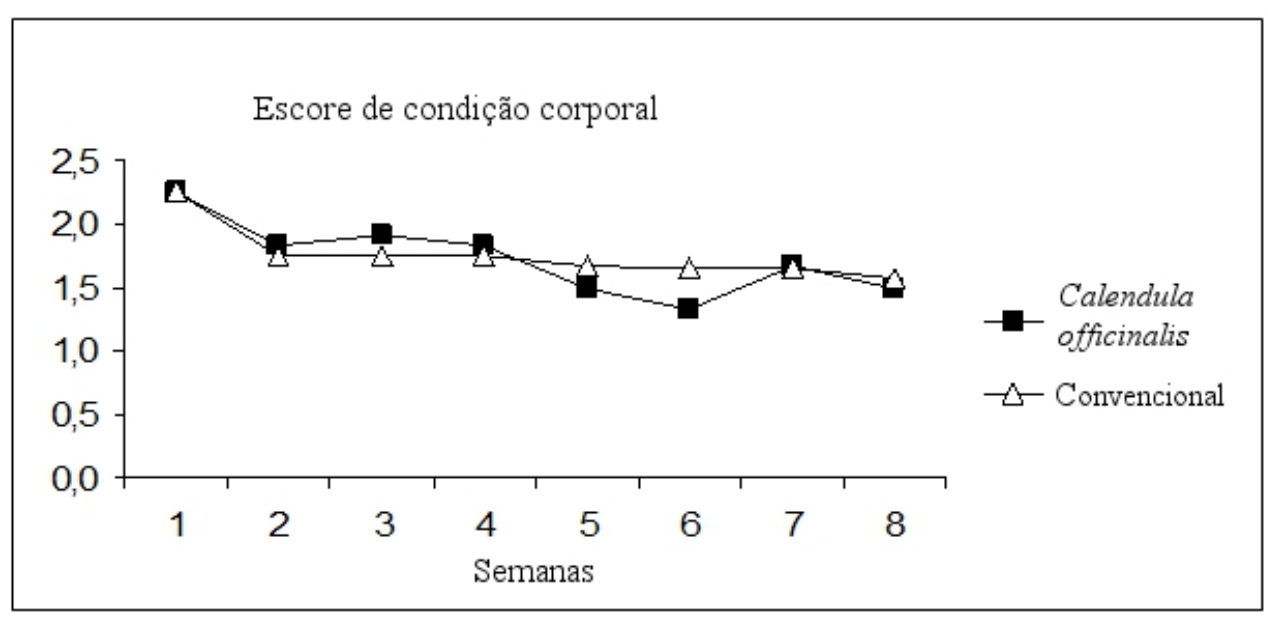

Figura 1. Escore de condição corporal (ECC) no decorrer do período experimental.

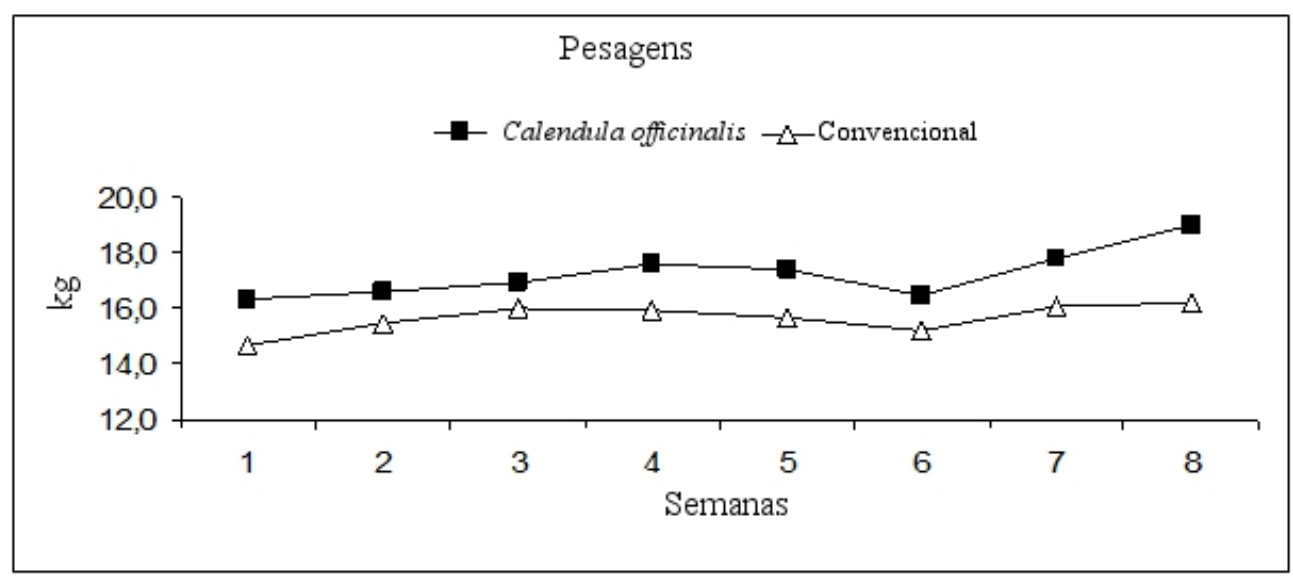

Figura 2. Ganho de peso no decorrer do período experimental.

Trabalhando com diferentes métodos de castração, BRAGA et al. (2003) observaram que os 
métodos cruentos podem causar estresse aos animais, demandando maior tempo para recuperação, provocando ligeira tendência de diminuição de ganho de peso dos animais.

Estudos têm correlacionado as medidas biométricas, principalmente as medidas de peso, altura de cernelha, altura de garupa, perímetro torácico, e o monitoramento do escore de condição corporal, para avaliar a conformação, aparência geral, desenvolvimento, peso vivo e para obter índices de rendimento de carcaça dos animais, de acordo com YÁÑEZ (2004).

De forma subjetiva, observou-se que os animais tratados com a pomada à base de Calendula officinalis tiveram menor reação dolorosa ao toque e menor formação de edema do que os tratados com o tratamento convencional.

A Calendula officinalis tem um efeito antiinflamatório tópico e efeito imunomodulador antiviral, estimulando atividade do sistema imune. $\mathrm{Na}$ Europa, pomadas à base de extratos de C. officinalis são usadas no tratamento de lesões orais, cortes e feridas e mostram efeito imunomodulatório e antibacteriano e ativam a regeneração tecidual e desenvolvimento de tecido epitelial (BLUMENTAL et al., 2000).

Estudos feitos com extrato aquoso de $C$. officinalis demonstraram um efeito de neovascularização, ajudando, portanto, na cicatrização. Estudos químicos feitos com esse extrato demostraram que os componentes predominantes são flavonóides e flavonóides glicosídicos, sendo estes, provavelmente, os responsáveis pelo efeito terapêutico (PATRICK et al., 1996). A C. officinalis contem vários componentes como triterpenóides e flavonóides glicosídicos, que são os principais constituintes com efeito antiinflamatório, além do ácido palmítico e da capsaicina, com forte ação analgésica e que, associados no extrato da planta, vão conferir por sinergismo o efeito de inibição da dor tanto neurogênica quanto inflamatória (VOLPATO, 2001).

Estudos evidenciaram que o uso de $C$. officinalis em uma formulação exclusiva é mais efetiva na reparação da pele lesionada do que seu uso em associação (VOLPATO, 2001), mas em caprinocultura isso se torna inviável devido à instalação de miíase nas feridas ser muito comum em regiões tropicais, por isso a associação com a Azadirachta indica, que vem se demonstrado importante no controle de insetos hematófagos devido ao largo espectro de ação e baixíssima toxicidade (COSTA et al., 2008), assim como o Cimbopogom nardus (BUENO et. al, 1999). A solução de própolis possui ação antimicrobiana in vivo (RAHAL et al, 2003), controlando a infecção da ferida e a cera participando como excipiente (CARVALHO, 2005).

Como os animais de produção, particularmente ruminantes, têm comportamento relativamente tranquilo, é possível que os mesmos não demonstrem sinais de estresse e dor de modo claro, levando a respostas fisiológicas complexas.

Além da dor aguda associada ao procedimento cirúrgico, o período pós-operatório também causa com sofrimento e a sensação de dor pode, inclusive, tornar-se crônica (DUNCAN, 2005). Portanto, é possível afirmar que o uso de medidas que amenizem o estresse e que promova uma rápida recuperação vão propiciar melhor bem-estar e volta às atividades fisiológicas normais de forma mais rápida.

\section{CONCLUSÕES}

O fitoterápico à base de Calendula offcinalis e associações pode ser usado no tratamento de ferimentos de animais de produção sem efeitos negativos quanto ao desempenho dos animais.

A pomada à base de extrato de Calendula officinalis, extrato de Azadiractha indica, extrato de Cimbopogom nardus, própolis e cera de abelha pode ser usada como alternativa ao uso de pomada à base de óxido de zinco, óleo de pinho, caulim e xilol e spray à base de permetrina, fenoxibenzil e DDVP.

\section{REFERÊNCIAS}

BLUMENTHAL, M.; GOLDBERG, A.; BRINCKMANN, J. Herbal Medicine. $1^{\mathrm{a}}$ edição. Newton M.: Integrative Medicine Communications, p. 44-45, 2000 .

BRAGA, Z. C. A. da C.; BRAGA, A. P.; VASCONCELOS, S. H. L. Efeito da Castração Sobre Ganho de Peso e Características da Carcaça de Caprinos SRD. Caatinga, v.16, b.1/2, p.13-15, 2003.

CARVALHO, D. M. O regimento contra a pestilência e a receita do bálsamo: alguns comentários à luz da "medicina científica". História, Ciências, Saúde - Manguinhos, Rio de Janeiro. v. 12, n. 3, p. 855-67, 2005.

CAMPOS, M. C. P. S.; COELHO, M. C. O. C.; SILVA, L. B. G.; MONTEIRO, V. L. C.; LIMA, E. T.; ACETO, M. L. Tratamento de Feridas Infectadas utilizando Calendula officinalis. Homeopatia Brasileira, v. 6, n. 1, p. 22-28, 2000.

COSTA, F. B.; VASCONCELOS, P. S. S.; SILVA, A. M. M., BRANDÃO, V. M., SILVA, I. A., TEIXEIRA, W. C.; GUERRA, R. M. S. N.; SANTOS, A. C. G. Eficácia de fitoterápicos em fêmeas ingurgitadas de Boophilus microplus, provenientes da mesorregião oeste do Maranhão, Brasil. Revista Brasileira de Parasitologia Veterinária. v.17, supl.1, p. 83-86, 2008. 
COSTA, M. G. USP/DT (Agência USP de Inovação). Serviço Brasileiro de Respostas Técnicas - SBRT, 2007. Disponível em: http://www.sbrt.ibict.br. Acesso em: 22/08/2007.

DUNCAN, I.J.H. Science-based assessment of animal welfare: farm animals. Revue Scientifique et Technique (International Office of Epizootics), v. 24, n. 2, p. 483492, 2005.

GUTERRES, S.; ZIEGLER, S. Matérias-primas vegetais com ação antiinflamatória empregadas em produtos dermo-cosméticos. Pharmacia Brasileira, v.30, p. 79-83, 2002.

LANS, C.; TURNER, N.; KHAN, T.; BRAUER, G.; BOEPPLE, W. Ethnoveterinary medicines used for ruminants in British Columbia, Canada. Journal of Ethnobiology and Ethnomedicine, v.3, p.11. 2007. Disponivel

em http://www.ethnobiomed.com/content/3/1/11, Acesso em 10 Jan 2012.

LOUCA, A.; ECONOMIDES, S. \& HANCOCK, J. Effects of Castration on Growth Rate, Feed Conversion Efficiency and Carcass Quality in Damascus Goats. Animal Production, vol. 24, p. 387-391, 1977.

MADRUGA, M. S.; ARRUDA, S. G. B. de; ANDRADE, L.T.; BESERRA, F. J. Efeito da castração sobre parâmetros químicos, físico-químicos e sensoriais da carne caprina de animais mestiços. Ciência e Tecnologia de Alimentos v. 20, n. 1, p. 23-26, 2000.

MARTINS, E. C.; GARAGORRY, F. L. A dinâmica das populações de caprinos no Brasil 12/12/2007. Disponível em:

www.portaldoagronegocio.com.br/conteudo.php?id=2367 5 Acesso em 06/08/2008.

MENEZES, J. J. L.; GONÇALVES, H. C.; RIBEIRO, M. S.; RODRIGUES, L.; CAÑIZARES, G. I. L.; MEDEIROS, B. B. L.; GIASSETTI, A. P. Desempenho e medidas biométricas de caprinos de diferentes grupos raciais. Revista Brasileira de Zootecnia, vol. 36, n. 3,p. 635-642, 2007.
MORAND-FEHR, P.; HERVIEU, J. Apprécier l'éat corporel des chèvres: Intérêt et méthod. Reussir La Chevre, n.231, p.22-34, 1999.

NORMAN, M. The potencial of meat from goat. Booker Tate Limited, Oxfordshire, UK, 1985.

OWEN, J. E.; CERECERES, M. T. A; MACIAS, J. A G.; GONZALEZ, F. A. N. Studies on the Criollo Goat of Nothern Mexico: Part 1- The effects of Body Weight on Body Components and Carcass Development. Meat Science, v. 9, p. 191-204, 1983.

PARK, Y. K.; IKEGAKI, M.; ABREU, J. A. S.; ALCICI, N. M. F. Estudo da Preparação dos Extratos de Própolis e suas Aplicações. Ciência e Tecnologia de Alimentos, v.18, n.1, p.313-318, 1998.

PATRI.CK, K.F.; KUMAR, S.; EDWARDSON, P.A., HUTCHINSON, J.J. Induction of vascularisation by an aqueous extract of the flowers of Calendula officinalis L. the European marigold. Phytomedicine, v.3, n.1, p.11-18, 1996.

RAHAL, S. C.; BRACARENSE, A. P. F. R. L.; TANAKA, C. Y.; GRILLO, T. P.; LEITE, C. A. L. Utilização de própolis ou mel no tratamento de feridas limpas induzidas em ratos. Archives of Veterinary Science. v. 8, n. 1, p. 61-67, 2003.

RIBEIRO, S. D. A. Caprinocultura: criação racional de caprinos. São Paulo: Editora Nobel, 1997.

SAEG - Sistema para Análises Estatísticas, Versão 9.1: Fundação Arthur Bernardes - UFV - Viçosa, 2007.

VOLPATO, A. M. M.; CECHINEL FILHO, V.; YUNES, R.; GOLIN, V.; SOUZA, M. M. Avaliação dos Efeitos Antinociceptivos do Extrato Hidroalcoólico obtido das Flores de Calendula officinalis. Alcance (UNIVALI), Itajaí, v. 8, n.5, p. 47-52, 2001.

YÁÑEZ, E. A.; RESENDE, K. T.; FERREIRA, A. C. D.; MEDEIROS, A. N.; SOBRINHO, A. G. S.; PEREIRA FILHO, J. M.; TEIXEIRA, I. A. M. A.; ARTONI, S. M. B. Utilização de Medidas Biométricas para Predizer Características da Carcaça de Cabritos Saanen. Revista Brasileira de Zootecnia. v.33, n.6, p.1564-1572, 2004 